\title{
Some remarks on the stability of the Cauchy equation and completeness
}

\author{
Harald Fripertinger@i And Jens Schwaiger
}

Unserem Lehrer, Kollegen und Freund Ludwig Reich nachträglich, aber mit den allerbesten Wünschen, zu seinem 80. Geburtstag gewidmet.

\begin{abstract}
It was proved in Forti and Schwaiger (C R Math Acad Sci Soc R Can 11(6):215-220, 1989), Schwaiger (Aequ Math 35:120-121, 1988) and with different methods in Schwaiger (Developments in functional equations and related topics. Selected papers based on the presentations at the 16th international conference on functional equations and inequalities, ICFEI, Będlewo, Poland, May 17-23, 2015, Springer, Cham, pp 275-295, 2017) that under the assumption that every function defined on suitable abelian semigroups with values in a normed space such that the norm of its Cauchy difference is bounded by a constant (function) is close to some additive function, i.e., the norm of the difference between the given function and that additive function is also bounded by a constant, the normed space must necessarily be complete. By Schwaiger (Ann Math Sil 34:151-163, 2020) this is also true in the nonarchimedean case. Here we discuss the situation when the bound is a suitable non-constant function.
\end{abstract}

Mathematics Subject Classification. 39B82, 46S10.

Keywords. Cauchy equation, Hyers-Ulam stability, Completeness.

\section{Introduction}

In [2, section 1] the author mentions that the original result by Hyers in [5] holds true for commutative semigroups in general. Thus we have the following result.

Theorem 1. (Forti and Hyers) Let $S$ be a commutative semigroup and $X$ be a Banach spaces and let $f: S \rightarrow X$ be a function such that for some $\varepsilon>0$

$$
\|f(x+y)-f(x)-f(y)\| \leq \varepsilon \text { for all } x, y \in S .
$$


Then for every $x \in S$ the limit $\alpha(x)=\lim _{n \rightarrow \infty} \frac{f\left(2^{n} x\right)}{2^{n}}$ exists, the function $\alpha$ is additive and

$$
\|f(x)-\alpha(x)\| \leq \varepsilon \quad \text { for all } x \in S .
$$

Moreover $\alpha$ is the unique additive function satisfying the last inequality.

In the proof it is shown that the sequence $\left(\frac{f\left(2^{n} x\right)}{2^{n}}\right)_{n \in \mathbb{N}_{0}}$ is a Cauchy sequence, and thus convergent, since $X$ is assumed to be a Banach space. But one might ask whether this assumption is necessary to find some additive $\alpha$ such that (2) holds.

It was shown in [3] that the completeness of $X$ is in some sense necessary.

Theorem 2. (Forti and Schwaiger) Let $G$ be an abelian group with an element of infinite order and $X$ a normed space. Assume that for all $f: G \rightarrow X$ and all $\varepsilon>0 \mathrm{Eq}$. (1) implies the existence of an additive function $\alpha: G \rightarrow X$ such that

$$
\|f(x)-\alpha(x)\| \leq \delta \text { for all } x \in G,
$$

for some $\delta>0$. Then $X$ is a Banach space.

For $G=\mathbb{Z}$ this has been proved earlier in [6] and for $G$ as in the above theorem by a different method in [7, Theorem 12.7].

Condition (1) has been weakened by replacing $\varepsilon$ by suitable functions $\varphi$ depending on $x$ and $y$ with the result that the bound in (2) also depends on $x, y$ and $\varphi$. Many of these results (see [2, pp. 9-11]) are special cases of a very general result (see [1]) for equations of the form $g[F(x, y)]=H[g(x), g(y)]$ with the bounding function $\varphi \geq 0$ such that $\sum_{i=1}^{\infty} 2^{-i} \varphi\left(2^{i-1} x, 2^{i-1} x\right)<\infty$ for all $x$ and $\lim _{i \rightarrow \infty} 2^{-i} \varphi\left(2^{i-1} x, 2^{i-1} x\right)=0$ for all $x, y$.

Recently LUDWIG REICH posed the question whether a result similar to Theorem 2 would hold true in this more general situation. In what follows this will be answered in the affirmative.

\section{A stability result}

The conditions from [1] are in the special case of the Cauchy equation contained in the conditions below when $p=2$.

Theorem 3. Let $X$ be a Banach space and $S$ an Abelian semigroup. Assume that $\varphi: S \times S \rightarrow[0, \infty)$ and $f: S \rightarrow X$ satisfy

$$
\|f(x+y)-f(x)-f(y)\| \leq \varphi(x, y), \quad x, y \in S .
$$

Let moreover $p \in \mathbb{N}$ be greater than 1 , put

$$
\varphi_{p}(x):=\sum_{j=1}^{p-1} \varphi(j x, x), \quad x \in S,
$$


and assume

(i) $\Phi_{p}(x):=\sum_{j=1}^{\infty} \frac{\varphi_{p}\left(p^{j-1} x\right)}{p^{j}}<\infty$ for all $x \in S$ and

(ii) $\lim _{n \rightarrow \infty} \frac{\varphi\left(p^{n} x, p^{n} y\right)}{p^{n}}=0$ for all $x, y \in S$.

Then there is an additive function $a: S \rightarrow X$ such that

$$
\|f(x)-a(x)\| \leq \Phi_{p}(x) \text { for all } x \in S .
$$

This $a$ is unique among all additive functions $b$ satisfying $\|f(x)-b(x)\| \leq$ $c \Phi_{p}(x)$ for all $x \in S$ (and some $c \geq 0$ depending possibly on $b$ ) and may be defined by $a(x)=\lim _{n \rightarrow \infty} \frac{f\left(p^{n} x\right)}{p^{n}}$.

Proof. (3) for $y=x$ implies $\|f(2 x)-2 f(x)\| \leq \varphi(x, x)$. Using induction on $q$ we get

$$
\|f(q x)-q f(x)\| \leq \varphi_{q}(x), \quad q \in \mathbb{N}, q>1, x \in S .
$$

In particular this holds true for $q=p$. Applying (6) to $p^{j-1} x, j \geq 1$, instead of $x$ yields

$$
\left\|f\left(p^{j} x\right)-p f\left(p^{j-1} x\right)\right\| \leq \varphi_{p}\left(p^{j-1} x\right)
$$

and

$$
\left\|f\left(p^{j} x\right) / p^{j}-f\left(p^{j-1} x\right) / p^{j-1}\right\| \leq \frac{\varphi_{p}\left(p^{j-1} x\right)}{p^{j}} .
$$

Writing $f_{j}(x):=f\left(p^{j} x\right) / p^{j}, x \in S, j \in \mathbb{N}_{0}$, the above equation reads as

$$
\left\|f_{j}(x)-f_{j-1}(x)\right\| \leq \frac{\varphi_{p}\left(p^{j-1} x\right)}{p^{j}}, \quad j \in \mathbb{N}, x \in S .
$$

This may be used to show that the sequence $\left(f_{j}(x)\right)_{j \in \mathbb{N}_{0}}$ is a Cauchy sequence since

$$
\begin{aligned}
\left\|f_{j+l}(x)-f_{j}(x)\right\| & \leq \sum_{k=1}^{l}\left\|f_{j+k}(x)-f_{j+k-1}(x)\right\| \\
& \leq \sum_{k=1}^{l} \frac{\varphi_{p}\left(p^{j+k-1} x\right)}{p^{j+k}} \leq \sum_{k=1}^{\infty} \frac{\varphi_{p}\left(p^{j+k-1} x\right)}{p^{j+k}} \\
& =\sum_{k=j+1}^{\infty} \frac{\varphi_{p}\left(p^{k-1} x\right)}{p^{k}}, \quad x \in S,
\end{aligned}
$$

and since the latter series is a tail of the series $\Phi_{p}(x)$ :

$$
\sum_{k=j+1}^{\infty} \frac{\varphi_{p}\left(p^{k-1} x\right)}{p^{k}}=\Phi_{p}(x)-\sum_{k=1}^{j} \frac{\varphi_{p}\left(p^{k-1} x\right)}{p^{k}} \rightarrow 0 \text { for } j \rightarrow \infty .
$$


Since $X$ is complete the function $a: S \rightarrow X, a(x):=\lim _{j \rightarrow \infty} f_{j}(x)$ is well defined. Moreover (10) for $j=0$ with $l \rightarrow \infty$ gives

$$
\|a(x)-f(x)\| \leq \sum_{k=1}^{\infty} \frac{\varphi_{p}\left(p^{k-1} x\right)}{p^{k}}=\Phi_{p}(x) .
$$

Moreover $a$ is additive: Since $S$ is Abelian we may estimate

$$
\begin{aligned}
\| f_{j}(x+y)- & f_{j}(x)-f_{j}(y)\left\|=\frac{1}{p^{j}}\right\| f\left(p^{j} x+p^{j} y\right)-f\left(p^{j} x\right)-f\left(p^{j} y\right) \| \\
\leq & \frac{\varphi\left(p^{j} x, p^{j} y\right)}{p^{j}} .
\end{aligned}
$$

For $j \rightarrow \infty$ we get by (ii) that $\|a(x+y)-a(x)-a(y)\|=0$ as desired.

Now let $b: S \rightarrow X$ be another additive function such that for some $c>0$ we have $\|f(x)-b(x)\| \leq c \Phi_{p}(x)$ for all $x \in S$. Then the additive function $d:=b-a$ satisfies $\|d(x)\| \leq(c+1) \Phi_{p}(x)$ for all $x$. Because of the additivity of $d$ substituting $p^{n} x$ for $x$ results in

$$
\begin{aligned}
p^{n}\|d(x)\| & =\left\|d\left(p^{n} x\right)\right\| \leq(c+1) \Phi_{p}\left(p^{n} x\right) \text { or } \\
\|d(x)\| \leq(c+1) \frac{\Phi_{p}\left(p^{n} x\right)}{p^{n}}=(c+1) \sum_{j=1}^{\infty} \frac{\varphi_{p}\left(p^{n+j-1} x\right)}{p^{n+j}} & \\
& =(c+1) \sum_{j=n+1}^{\infty} \frac{\varphi_{p}\left(p^{j-1} x\right)}{p^{j}},
\end{aligned}
$$

which tends to 0 for $n \rightarrow \infty$ by (11). Thus $d(x)=0$ for all $x$, i. e., $a=b$.

Remark 1. 1. As mentioned before the case $p=2$ results in a well known result (see [1] and additionally the references in [2] and also [4]). For $p>2$ the result seems to be new in the archimedean case. For stability investigations where the target space of the function involved is a Banach space over the non archimedean valued field $\mathbb{Q}_{p}$ some results may be found in [8]. But of course the question arises whether there are functions $\varphi$ such that $\varphi_{p}$ satisfies the conditions of the theorem for some $p>2$, but $\varphi_{2}$ does not. An example of such $\varphi$ is, for example, the following. Let $S:=\mathbb{N}$ and define $\varphi: S \times S \rightarrow[0, \infty)$ by $\varphi(x, y):=1$ if $3 \mid x$ and $3 \mid y$, $\varphi\left(2^{n}, 2^{m}\right):=2^{n+m}$ for all $n, m \in \mathbb{N}$, and let $\varphi(x, y)$ be arbitrary in all the remaining cases. Then the conditions (i) and (ii) are satisfied for all $x, y$ if $p=3$. But (ii) and thus also (i) is not satisfied for $p=2$ since $\varphi_{2}\left(2^{n} \cdot 1,2^{n} \cdot 1\right) / 2^{n}=2^{n}$ for all $n$.

2. Obviously the Theorem may be applied in the particular case when $\varphi=$ $\varepsilon>0$ is constant. 


\section{Some characterizations of completeness by stability, the archimedean case}

Consider an integer $p>1$. Let $S$ be a commutative semigroup and put $\mathcal{F}:=\mathcal{F}_{p}:=\{\varphi: S \times S \rightarrow[0, \infty) \mid \varphi$ satisfies (i) and (ii) of Theorem 3$\}$. For $\varphi \in \mathcal{F}$ define $\Phi=\Phi_{\varphi}: S \rightarrow[0, \infty)$ by

$$
\Phi(x):=\sum_{n=1}^{\infty} \frac{\varphi_{p}\left(p^{n-1} x\right)}{p^{n}}<\infty, \quad x \in S,
$$

where $\varphi_{p}$ is defined by (4). If for $m \geq 1$ we denote by $\Phi_{\varphi, m}(x)$ the tail

$$
\Phi_{\varphi, m}(x):=\sum_{n=m}^{\infty} \frac{\varphi_{p}\left(p^{n-1} x\right)}{p^{n}}<\infty,
$$

then, as mentioned in (11), $\lim _{m \rightarrow \infty} \Phi_{\varphi, m}(x)=0$.

Let $\mathcal{K} \subseteq \mathcal{F}$ be a non-empty cone. I.e., $\mathcal{K}+\mathcal{K} \subseteq \mathcal{K},[0, \infty) \cdot \mathcal{K} \subseteq \mathcal{K}$ (and $\mathcal{K} \neq \emptyset$ ). If $X$ is a normed space over $\mathbb{Q}$ with completion $X^{c}$, then the set $\mathcal{C}:=\mathcal{C}_{\mathcal{K}}=\left\{f \in X^{S} \mid \exists \varphi \in \mathcal{K}:\|f(x+y)-f(x)-f(y)\| \leq \varphi(x, y), x, y \in S\right\}$ is a subspace of $X^{S}$. In the sequel we will indicate the Cauchy difference $f(x+y)-f(x)-f(y)$ by $\gamma_{f}(x, y)$. Motivated by the proof of Theorem 3 and certain results of [7] let

$$
\alpha: \mathcal{C} \rightarrow \operatorname{Hom}\left(S, X^{c}\right), \quad \alpha(f)(x):=\lim _{n \rightarrow \infty} \frac{f\left(p^{n} x\right)}{p^{n}} .
$$

This mapping is well defined and linear.

Theorem 4. Using the notation from above, $\operatorname{ker}(\alpha)$ is equal to

$$
\left\{f \in X^{S} \mid \exists \varphi \in \mathcal{K}:\left\|\gamma_{f}(x, y)\right\| \leq \varphi(x, y) \text { and }\|f(x)\| \leq \Phi_{\varphi}(x), x, y \in S\right\} .
$$

Proof. If $f$ belongs to the kernel of $\alpha$, then $\|f(x)\|=\|f(x)-\alpha(f)(x)\| \leq$ $\Phi_{\varphi}(x), x \in S$, by an application of Theorem 3 .

Conversely, if $f$ belongs to $\mathcal{C}_{\mathcal{K}}$, then

$$
\left\|\frac{f\left(p^{n} x\right)}{p^{n}}\right\| \leq \frac{\Phi_{\varphi}\left(p^{n} x\right)}{p^{n}}=\sum_{j=1}^{\infty} \frac{\varphi_{p}\left(p^{j-1} p^{n} x\right)}{p^{j} p^{n}}=\Phi_{\varphi, n+1}(x) \underset{n \rightarrow \infty}{\rightarrow} 0 \text { by (11), }
$$

thus $\alpha(f)=0$.

To each $\varphi \in \mathcal{F}$ we associate some $\psi=\psi_{\varphi}: S \times S \rightarrow[0, \infty)$ defined by

$$
\psi(x, y)=\varphi(x+y, x+y)+\varphi(x, x)+\varphi(y, y) .
$$

Theorem 5. If there exists some $\varphi \in \mathcal{K}$ such that $\psi=\psi_{\varphi}$ belongs to $\mathcal{K}$, and if $\varphi(x, x)>0$ for all $x \in S$, then $\alpha$ is surjective. 
Proof. Consider some $a \in \operatorname{Hom}\left(S, X^{c}\right)$ and some $\varphi \in \mathcal{K}$ such that $\psi \in \mathcal{K}$ and $\varphi(x, x)>0$ for all $x \in S$. Since $\bar{X}=X^{c}$ we may find for each $x \in$ $S$ some value $f(x) \in X$ such that $\|f(x)-a(x)\|<\varphi(x, x)$. This defines a function $f: S \rightarrow X$. Then $\gamma_{f}(x, y)=(f(x+y, x+y)-a(x+y))-(f(x, x)-$ $a(x))-(f(y, y)-a(y))$ and, therefore, $\left\|\gamma_{f}(x, y)\right\| \leq \varphi(x+y, x+y)+\varphi(x, x)+$ $\varphi(y, y)=\psi(x, y)$. From $\psi \in \mathcal{K}$ we derive that $f \in \mathcal{C}$. By Theorem 3 there exists a unique $b \in \operatorname{Hom}\left(S, X^{c}\right)$ such that $\|f-b\| \leq \Phi_{\psi}$. It is given by $b(x)=$ $\lim _{n \rightarrow \infty} f\left(p^{n} x\right) / p^{n}=\alpha(f)(x), x \in S$.

Moreover $\left\|f\left(p^{n} x\right)-p^{n} a(x)\right\|=\left\|f\left(p^{n} x\right)-a\left(p^{n} x\right)\right\| \leq \varphi\left(p^{n} x, p^{n} x\right), x \in S$, thus

$$
\left\|\frac{f\left(p^{n} x\right)}{p^{n}}-a(x)\right\| \leq \frac{\varphi\left(p^{n} x, p^{n} x\right)}{p^{n}} \underset{n \rightarrow \infty}{\rightarrow} 0, \quad x \in S,
$$

by (ii) of Theorem 3. Consequently $\|b(x)-a(x)\|=0$, thus $a=b=\alpha(f)$, and $a$ belongs to the image of $\alpha$.

Remark 2. Using the notation from above we have

$$
\mathcal{C} / \operatorname{Ker}(\alpha) \cong \operatorname{Hom}\left(S, X^{c}\right)
$$

provided that there exists some $\varphi \in \mathcal{K}$ such that $\psi=\psi_{\varphi}$ belongs to $\mathcal{K}$ and that $\varphi(x, x)>0$ for all $x \in S$.

Theorem 6. For each $\varphi \in \mathcal{F}$ the function $\psi=\psi_{\varphi}$ belongs to $\mathcal{F}$.

Proof. Let $\varphi \in \mathcal{F}$ and $N \in \mathbb{N}$. Then $\psi$ satisfies (i) since

$$
\begin{aligned}
& \sum_{n=1}^{N} \frac{\psi_{p}\left(p^{n-1} x\right)}{p^{n}}=\sum_{n=1}^{N} \frac{1}{p^{n}} \sum_{j=1}^{p-1} \psi\left(j p^{n-1} x, p^{n-1} x\right) \\
& =\sum_{n=1}^{N} \frac{1}{p^{n}} \sum_{j=1}^{p-1}\left(\varphi\left((j+1) p^{n-1} x,(j+1) p^{n-1} x\right)+\varphi\left(j p^{n-1} x, j p^{n-1} x\right)+\varphi\left(p^{n-1} x, p^{n-1} x\right)\right) \\
& \leq \sum_{n=1}^{N} \frac{1}{p^{n}} \sum_{j=1}^{p-1} \sum_{k=1}^{p-1}\left(\varphi\left(k(j+1) p^{n-1} x,(j+1) p^{n-1} x\right)+\varphi\left(k j p^{n-1} x, j p^{n-1} x\right)\right. \\
& \left.\quad \quad+\varphi\left(k p^{n-1} x, p^{n-1} x\right)\right) \\
& =\sum_{j=1}^{p-1} \sum_{n=1}^{N} \frac{1}{p^{n}}\left(\varphi_{p}\left((j+1) p^{n-1} x\right)+\varphi_{p}\left(j p^{n-1} x\right)+\varphi_{p}\left(p^{n-1} x\right)\right) \\
& \leq \sum_{j=1}^{p-1}\left(\Phi\left((j+1) p^{n-1} x\right)+\Phi\left(j p^{n-1} x\right)+\Phi\left(p^{n-1} x\right)\right)<\infty,
\end{aligned}
$$

and (ii) since

$$
\frac{\psi\left(p^{n} x, p^{n} y\right)}{p^{n}}=\frac{\varphi\left(p^{n}(x+y), p^{n}(x+y)\right)+\varphi\left(p^{n} x, p^{n} x\right)+\varphi\left(p^{n} y, p^{n} y\right)}{p^{n}} \underset{n \rightarrow \infty}{\rightarrow} 0
$$

which finishes the proof. 
Now we can characterize completeness of $X$ in the following way:

Theorem 7. Consider $S=\mathbb{N}_{0}$, an integer $p>1, X$ a normed space over $\mathbb{Q}$, and $\varphi \in \mathcal{F}=\mathcal{F}_{p}$ satisfying $\varphi(n, n)>0$ for all $n \in \mathbb{N}_{0}$.

If for each function $f: \mathbb{N}_{0} \rightarrow X$ satisfying

$$
\left\|\gamma_{f}(n, m)\right\| \leq \psi_{\varphi}(n, m), \quad n, m \in \mathbb{N}_{0},
$$

there exists some $b \in \operatorname{Hom}\left(\mathbb{N}_{0}, X\right)$ such that $\|f-b\| \leq \Phi_{\psi}, \psi=\psi_{\varphi}$, then $X=X^{c}$, thus $X$ is complete.

Proof. Consider some $x_{0} \in X^{c}$, an element of the closure of $X, \varphi \in \mathcal{F}$ satisfying $\varphi(n, n)>0$ for all $n \in \mathbb{N}_{0}$, and $a \in \operatorname{Hom}\left(\mathbb{N}_{0}, X^{c}\right)$ given by $a(n)=n x_{0}$. For each $n \in \mathbb{N}_{0}$ there exists a value $f(n) \in X$ such that $\|f(n)-a(n)\|<\varphi(n, n)$. This defines a function $f \in X^{\mathbb{N}_{0}}$. Then $\left\|\gamma_{f}(n, m)\right\| \leq\|f(n+m)-a(n+m)\|+$ $\|f(n)-a(n)\|+\|f(m)-a(m)\| \leq \varphi(n+m, n+m)+\varphi(n, n)+\varphi(m, m)=$ $\psi_{\varphi}(n, m), n, m \in \mathbb{N}_{0}$. Theorem 6 implies $\psi_{\varphi} \in \mathcal{F}$, therefore $\alpha$ : $\mathcal{C}_{\mathcal{F}} \rightarrow \operatorname{Hom}\left(\mathbb{N}_{0}, X^{c}\right)$ is surjective. By assumption, there exists some $b \in \operatorname{Hom}\left(\mathbb{N}_{0}, X\right)$ such that $\|f(n)-b(n)\| \leq \Phi_{\psi}(n), n \in \mathbb{N}_{0}$. Let $\xi=b(1)$, then $b(n)=n \cdot b(1)=n \xi$, whence,

$n\left\|\xi-x_{0}\right\|=\left\|n \xi-n x_{0}\right\| \leq\|n \xi-f(n)\|+\left\|f(n)-n x_{0}\right\| \leq \Phi_{\psi}(n)+\varphi(n, n), \quad n \in \mathbb{N}_{0}$.

For $n=p^{m}, m \in \mathbb{N}_{0}$, we obtain

$$
\left\|\xi-x_{0}\right\| \leq \frac{1}{p^{m}}\left(\Phi_{\psi}\left(p^{m}\right)+\varphi\left(p^{m}, p^{m}\right)\right)
$$

which by Theorem 5 and (ii) from Theorem 3 tends to 0 for $m \rightarrow \infty$. Hence, $\left\|\xi-x_{0}\right\|=0$ which means $x_{0}=\xi=b(1) \in X$, whence $X^{c} \subseteq X$ and $X=X^{c}$.

Corollary 1. Consider $S=\mathbb{N}_{0}$, an integer $p>1, X$ a normed space over $\mathbb{Q}$, and $\varepsilon>0$. If for each function $f: \mathbb{N}_{0} \rightarrow X$ satisfying

$$
\left\|\gamma_{f}(n, m)\right\| \leq \varepsilon, \quad n, m \in \mathbb{N}_{0},
$$

there exists some $b \in \operatorname{Hom}\left(\mathbb{N}_{0}, X\right)$ such that $\|f-b\| \leq \varepsilon$, then $X=X^{c}$, thus $X$ is complete.

Proof. This is the special case of Theorem 7 for $\varphi=\varepsilon / 3$ since then $\psi=\psi_{\varphi}=\varepsilon$, $\psi_{p}=(p-1) \varepsilon$, and $\Phi_{\psi}=(p-1) \varepsilon \sum_{n=1}^{\infty} p^{-n}=\varepsilon$.

\section{Some characterizations of completeness by stability, the non-archimedean case}

In a similar way the situation of non-archimedean normed spaces can be studied. 
In [8] the following theorem can be found. It is a reformulation of Theorem 3 .

Theorem 8. Let $X$ be a Banach space over $\left(\mathbb{Q},||_{p}\right)$, $p$ a prime, and $S$ an Abelian semigroup which is uniquely divisible by $p$, i.e., the mapping $S \ni x \mapsto$ $p x=: \pi(x) \in S$ is bijective. Assume that $\varphi: S \times S \rightarrow[0, \infty)$ and $f: S \rightarrow X$ satisfy

$$
\|f(x+y)-f(x)-f(y)\| \leq \varphi(x, y), \quad x, y \in S,
$$

together with

(i) $\Phi_{p}(x):=\sum_{n=1}^{\infty} \frac{1}{p^{n-1}} \varphi_{p}\left(\frac{x}{p^{n}}\right)<\infty$ for all $x \in S$ and

(ii) $\lim _{n \rightarrow \infty} \frac{\varphi\left(\frac{x}{p^{n}}, \frac{y}{p^{n}}\right)}{p^{n}}=0$ for all $x, y \in S$, where

$$
\varphi_{p}(x):=\sum_{j=1}^{p-1} \varphi(j x, x)
$$

and $x / p^{n}=\pi^{-n}(x)$.

Then there is an additive function $a: S \rightarrow X$ such that

$$
\|f(x)-a(x)\| \leq \Phi_{p}(x) \text { for all } x \in S .
$$

This a is unique among all additive functions b satisfying $\|f(x)-b(x)\| \leq$ $c \Phi_{p}(x)$ for all $x \in S$ (and some $c \geq 0$ depending possibly on $b$ ) and may be defined by $a(x)=\lim _{n \rightarrow \infty} p^{n} f\left(\frac{x}{p^{n}}\right)$.

Now let

$$
\mathcal{F}:=\mathcal{F}_{p}:=\{\varphi: S \times S \rightarrow[0, \infty) \mid \varphi \text { satisfies (i) and (ii) of Theorem } 8\} .
$$

For $\varphi \in \mathcal{F}$ define $\Phi=\Phi_{\varphi}: S \rightarrow[0, \infty)$ by

$$
\Phi(x):=\sum_{n=1}^{\infty} \frac{1}{p^{n-1}} \varphi_{p}\left(\frac{x}{p^{n}}\right)<\infty, \quad x \in S,
$$

where $\varphi_{p}$ is given by (14). If for $m \geq 1$ we denote by $\Phi_{\varphi, m}(x)$ the tail

$$
\Phi_{\varphi, m}(x):=\sum_{n=m}^{\infty} \frac{1}{p^{n-1}} \varphi_{p}\left(\frac{x}{p^{n}}\right)<\infty,
$$

and again $\lim _{m \rightarrow \infty} \Phi_{\varphi, m}(x)=0$.

Let $\mathcal{K} \subseteq \mathcal{F}$ be a non-empty cone. If $X$ is a normed space over $\left(\mathbb{Q},||_{p}\right), p$ a prime, with completion $X^{c}$, and $S$ is an Abelian semigroup which is uniquely divisible by $p$, then the set

$\mathcal{C}:=\mathcal{C}_{\mathcal{K}}=\left\{f \in X^{S} \mid \exists \varphi \in \mathcal{K}:\|f(x+y)-f(x)-f(y)\| \leq \varphi(x, y), x, y \in S\right\}$

is a subspace of $X^{S}$. Motivated by the proof of Theorem 8 let

$$
\alpha: \mathcal{C} \rightarrow \operatorname{Hom}\left(S, X^{c}\right), \quad \alpha(f)(x):=\lim _{n \rightarrow \infty} p^{n} f\left(\frac{x}{p^{n}}\right) .
$$


This mapping is well defined and linear.

Theorem 9. Using the notation from above

$\operatorname{Ker}(\alpha)=\left\{f \in X^{S} \mid \exists \varphi \in \mathcal{K}:\left\|\gamma_{f}(x, y)\right\| \leq \varphi(x, y)\right.$ and $\left.\|f(x)\| \leq \Phi_{\varphi}(x), x, y \in S\right\}$.

Proof. If $f$ belongs to the kernel of $\alpha$, then $\|f(x)\|=\|f(x)-\alpha(f)(x)\| \leq$ $\Phi_{\varphi}(x), x \in S$, by an application of Theorem 8 .

Conversely, if $f$ belongs to $\mathcal{C}_{\mathcal{K}}$, then

$$
\left\|p^{n} f\left(\frac{x}{p^{n}}\right)\right\|=\left|p^{n}\right|_{p}\left\|f\left(\frac{x}{p^{n}}\right)\right\| \leq p^{-n} \Phi_{\varphi}\left(\frac{x}{p^{n}}\right)=\Phi_{\varphi, n+m}(x) \underset{n \rightarrow \infty}{\rightarrow} 0,
$$

thus $\alpha(f)=0$.

Theorem 10. If there exists some $\varphi \in \mathcal{K}$ such that $\psi=\psi_{\varphi}$ belongs to $\mathcal{K}$, and if $\varphi(x, x)>0$ for all $x \in S$, then $\alpha$ is surjective.

Proof. Consider some $a \in \operatorname{Hom}\left(S, X^{c}\right)$ and some $\varphi \in \mathcal{K}$ such that $\psi \in \mathcal{K}$ and $\varphi(x, x)>0$ for all $x \in S$. Since $\bar{X}=X^{c}$ for each $x \in S$ we may find a value $f(x) \in X$ such that $\|f(x)-a(x)\|<\varphi(x, x)$. This defines a function $f: S \rightarrow$ $X$. Then $\gamma_{f}(x, y)=(f(x+y, x+y)-a(x+y))-(f(x, x)-a(x))-(f(y, y)-a(y))$ and therefore $\left\|\gamma_{f}(x, y)\right\| \leq \varphi(x+y, x+y)+\varphi(x, x)+\varphi(y, y)=\psi(x, y)$. From $\psi \in$ $\mathcal{K}$ we derive that $f \in \mathcal{C}$. By Theorem 8 there exists a unique $b \in \operatorname{Hom}\left(S, X^{c}\right)$ such that $\|f-b\| \leq \Phi_{\psi}$. It is given by $b(x)=\lim _{n \rightarrow \infty} p^{n} f\left(x / p^{n}\right)=\alpha(f)(x)$, $x \in S$.

Moreover, since $p^{n} a\left(\frac{x}{p^{n}}\right)=a(x), x \in S$, we have

$$
\left\|f\left(\frac{x}{p^{n}}\right)-p^{-n} a(x)\right\|=\left\|f\left(\frac{x}{p^{n}}\right)-a\left(\frac{x}{p^{n}}\right)\right\| \leq \varphi\left(\frac{x}{p^{n}}, \frac{x}{p^{n}}\right), \quad x \in S,
$$

thus

$$
\begin{aligned}
& \left\|p^{n} f\left(\frac{x}{p^{n}}\right)-a(x)\right\|=\left\|p^{n}\left(f\left(\frac{x}{p^{n}}\right)-p^{-n} a(x)\right)\right\|=\left|p^{n}\right|_{p}\left\|f\left(\frac{x}{p^{n}}\right)-p^{-n} a(x)\right\|= \\
& p^{-n}\left\|f\left(\frac{x}{p^{n}}\right)-a\left(\frac{x}{p^{n}}\right)\right\| \leq \varphi\left(\frac{x}{p^{n}}, \frac{x}{p^{n}}\right) / p^{n} \underset{n \rightarrow \infty}{\rightarrow} 0, \quad x \in S,
\end{aligned}
$$

by (ii) of Theorem 8 . Consequently $\|b(x)-a(x)\|=0$, thus $a=b=\alpha(f)$, and $a$ belongs to the image of $\alpha$.

Remark 3. Using the notation from above we have

$$
\mathcal{C} / \operatorname{Ker}(\alpha) \cong \operatorname{Hom}\left(S, X^{c}\right)
$$

provided that there exists some $\varphi \in \mathcal{K}$ such that $\psi=\psi_{\varphi}$ belongs to $\mathcal{K}$ and that $\varphi(x, x)>0$ for all $x \in S$.

Theorem 11. For each $\varphi \in \mathcal{F}$ the function $\psi=\psi_{\varphi}$ belongs to $\mathcal{F}$. 
Proof. We have to prove, that $\psi$ satisfies both (i) and (ii) from Theorem 8.

Let $N \in \mathbb{N}$ and $x \in S$. Then

$$
\begin{aligned}
& \sum_{n=1}^{N} \frac{1}{p^{n-1}} \psi_{p}\left(\frac{x}{p^{n}}\right)=\sum_{n=1}^{N} \frac{1}{p^{n-1}} \sum_{j=1}^{p-1} \psi\left(j \frac{x}{p^{n}}, \frac{x}{p^{n}}\right)= \\
& \sum_{n=1}^{N} \frac{1}{p^{n-1}} \sum_{j=1}^{p-1}\left(\varphi\left((j+1) \frac{x}{p^{n}},(j+1) \frac{x}{p^{n}}\right)+\varphi\left(j \frac{x}{p^{n}}, j \frac{x}{p^{n}}\right)+\varphi\left(\frac{x}{p^{n}}, \frac{x}{p^{n}}\right)\right) \leq \\
& \sum_{n=1}^{N} \frac{1}{p^{n-1}} \sum_{j=1}^{p-1} \sum_{k=1}^{p-1}\left(\varphi\left(k(j+1) \frac{x}{p^{n}},(j+1) \frac{x}{p^{n}}\right)+\varphi\left(k j \frac{x}{p^{n}}, j \frac{x}{p^{n}}\right)+\varphi\left(k \frac{x}{p^{n}}, \frac{x}{p^{n}}\right)\right)= \\
& \sum_{j=1}^{p-1} \sum_{n=1}^{N} \frac{1}{p^{n-1}}\left(\varphi_{p}\left((j+1) \frac{x}{p^{n}}\right)+\varphi_{p}\left(j \frac{x}{p^{n}}\right)+\varphi_{p}\left(\frac{x}{p^{n}}\right)\right) \leq \\
& \sum_{j=1}^{p-1}(\Phi((j+1) x)+\Phi(j x)+\Phi(x))<\infty .
\end{aligned}
$$

This holds true for all $N$, thus (i) is satisfied.

Let $x, y \in S$, then

$$
\frac{\psi\left(\frac{x}{p^{n}}, \frac{y}{p^{n}}\right)}{p^{n}}=\frac{\varphi\left(\frac{x+y}{p^{n}}, \frac{x+y}{p^{n}}\right)+\varphi\left(\frac{x}{p^{n}}, \frac{x}{p^{n}}\right)+\varphi\left(\frac{y}{p^{n}}, \frac{y}{p^{n}}\right)}{p^{n}} \underset{n \rightarrow \infty}{\rightarrow} 0
$$

which proves (ii).

Now we can characterize completeness of $X$ in the following way:

Theorem 12. Let $p$ be a prime. Consider $S=\left\{n p^{z} \mid n \in \mathbb{N}_{0}, z \in \mathbb{Z}\right\}, X$ a normed space over $\left(\mathbb{Q},||_{p}\right)$, and $\varphi \in \mathcal{F}_{p}$ satisfying $\varphi(x, x)>0$ for all $x \in S$. If for each function $f: S \rightarrow X$ such that

$$
\left\|\gamma_{f}(s, t)\right\| \leq \psi_{\varphi}(s, t), \quad s, t \in S,
$$

there exists some $b \in \operatorname{Hom}(S, X)$ such that $\|f-b\| \leq \Phi_{\psi}, \psi=\psi_{\varphi}$, then $X=X^{c}$, thus $X$ is complete.

Proof. Consider some $x_{0} \in X^{c}$, an element of the closure of $X, \varphi \in \mathcal{F}$ satisfying $\varphi(s, s)>0$ for all $s \in S$, and $a \in \operatorname{Hom}\left(S, X^{c}\right)$ given by $a(s)=s x_{0}$. For each $s \in$ $S$ there exists a value $f(s) \in X$ such that $\|f(s)-a(s)\|<\varphi(s, s)$. This defines a function $f \in X^{S}$. Then $\left\|\gamma_{f}(s, t)\right\| \leq\|f(s+t)-a(s+t)\|+\|f(s)-a(s)\|+$ $\|f(t)-a(t)\| \leq \varphi(s+t, s+t)+\varphi(s, s)+\varphi(t, t)=\psi_{\varphi}(s, t), s, t \in S$. Theorem 11 implies $\psi_{\varphi} \in \mathcal{F}$, therefore $\alpha: \mathcal{C}_{\mathcal{F}} \rightarrow \operatorname{Hom}\left(S, X^{c}\right)$ is surjective. By assumption, there exists some $b \in \operatorname{Hom}(S, X)$ such that $\|f(s)-b(s)\| \leq \Phi_{\psi}(s), s \in S$. Let 
$\xi=b(1)$, then $b\left(n p^{z}\right)=n p^{z} \cdot b(1)=n p^{z} \xi, n \in \mathbb{N}_{0}, z \in \mathbb{Z}$, whence,

$$
\begin{aligned}
\left|n p^{z}\right|_{p}\left\|\xi-x_{0}\right\|= & \left\|n p^{z} \xi-n p^{z} x_{0}\right\| \leq\left\|n p^{z} \xi-f\left(n p^{z}\right)\right\|+\left\|f\left(n p^{z}\right)-n p^{z} x_{0}\right\| \leq \\
& \Phi_{\psi}\left(n p^{z}\right)+\varphi\left(n p^{z}, n p^{z}\right), \quad n p^{z} \in S .
\end{aligned}
$$

For $n p^{z}=p^{-m}, m \in \mathbb{N}_{0}$ we obtain $\left|n p^{z}\right|_{p}=p^{m}$, and

$$
\left\|\xi-x_{0}\right\| \leq \frac{1}{p^{m}}\left(\Phi_{\psi}\left(\frac{1}{p^{m}}\right)+\varphi\left(\frac{1}{p^{m}}, \frac{1}{p^{m}}\right)\right)
$$

which by Theorem 9 and (ii) from Theorem 8 tends to 0 for $m \rightarrow \infty$. Hence, $\left\|\xi-x_{0}\right\|=0$ which means $x_{0}=\xi=b(1) \in X$, whence $X^{c} \subseteq X$ and $X=X^{c}$.

Corollary 2. Consider a prime $p, S$ as in Theorem 12, X a normed space over $\left(\mathbb{Q},||_{p}\right)$, and $\varepsilon>0$.

If for each function $f: S \rightarrow X$ satisfying

$$
\left\|\gamma_{f}(s, t)\right\| \leq \varepsilon, \quad s, t \in S,
$$

there exists some $b \in \operatorname{Hom}(S, X)$ such that $\|f-b\| \leq p \varepsilon$, then $X=X^{c}$, thus $X$ is complete.

Proof. This is the special case of Theorem 12 for $\varphi=\varepsilon / 3$ since then $\psi=\psi_{\varphi}=$ $\varepsilon, \psi_{p}=(p-1) \varepsilon$, and $\Phi_{\psi}=(p-1) \varepsilon \sum_{n=1}^{\infty} p^{-(n-1)}=p \varepsilon$.

Remark 4. Theorem 12 holds true also in the case when $S=\left\{n p^{z} \mid n \in\right.$ $\left.\mathbb{N}_{0}, z \in \mathbb{Z}\right\}$ is replaced by $S^{\prime}=\mathbb{Q}$ since in this case as well the additive functions defined on $S^{\prime}$ are determined uniquely by their values at 1 .

Remark 5. The Hyers sequence $\left(p^{n} f\left(x / p^{n}\right)\right)_{n \in \mathbb{N}_{0}}$ plays an important role when constructing an additive function close to $f$. Now it may be asked whether the sequence $\left(q^{n} f\left(x / q^{n}\right)\right)_{n \in \mathbb{N}_{0}}$ also converges when $q \neq p$.

First we analyze the consequences of the fact that $\left(q^{n} f\left(x / q^{n}\right)\right)_{n \in \mathbb{N}_{0}}$ converges when $p$ and $q$ are relatively prime. Let $p$ be a prime, $S$ an Abelian semigroup which is uniquely divisible by $p$, and $q$ be an integer coprime with $p$. Moreover assume that $\varphi: S \times S \rightarrow[0, \infty)$ and $f: S \rightarrow X, X$ a Banach space over $\left(\mathbb{Q},||_{p}\right)$, satisfy $\gamma_{f}(x, y) \leq \varphi(x, y), x, y \in S$. From the proof of Theorem 8 we get in a similar way as (9) and (10) in the proof of Theorem 3 that

$$
\begin{aligned}
\left\|q^{n+1} f\left(\frac{x}{q^{n+1}}\right)-q^{n} f\left(\frac{x}{q^{n}}\right)\right\| & =\left|q^{n}\right|_{p}\left\|f\left(q \frac{x}{q^{n+1}}\right)-q f\left(\frac{x}{q^{n+1}}\right)\right\| \\
& \leq \sum_{j=1}^{q-1} \varphi\left(j \frac{x}{q^{n+1}}, \frac{x}{q^{n+1}}\right)=: \varphi_{q}\left(\frac{x}{q^{n+1}}\right)
\end{aligned}
$$

and

$$
\left\|q^{n+m} f\left(\frac{x}{q^{n+1}}\right)-q^{n} f\left(\frac{x}{q^{n}}\right)\right\| \leq \sum_{j=1}^{m} \varphi_{q}\left(\frac{x}{q^{n+j}}\right) .
$$


If $\left(q^{n} f\left(x / q^{n}\right)\right)_{n \in \mathbb{N}_{0}}$ converges, then it is a Cauchy-sequence. In terms of $\varphi$ this can be guaranteed for instance if $\sum_{n=1}^{\infty} \varphi_{q}\left(\frac{x}{q^{n}}\right)<\infty$ which is much stronger than property (i) in Theorem 8.

In what follows next we give an example of a function $f: \mathbb{Q} \rightarrow \mathbb{Q}_{p}$ and a function $\varphi: \mathbb{Q} \times \mathbb{Q} \rightarrow[0, \infty)$ satisfying (i) and (ii) from Theorem 8 for some prime $p$ and also for $q>1$ coprime with $p$ such that

$$
\begin{aligned}
& \lim _{n \rightarrow \infty} p^{n} f\left(x / p^{n}\right)=0 \text { for all } x \in \mathbb{Q} \text { but } \\
& \left(q^{n} f\left(x / q^{n}\right)\right)_{n \in \mathbb{N}} \text { divergent for all } x \in \mathbb{Q} \backslash\{0\} .
\end{aligned}
$$

$f$ is defined by $f(0):=0$ and for $x \neq 0$ with $|x|_{p}=p^{m}$ by $f(x):=p^{-m / 2}$ if $m$ is even and by $f(x):=p^{-(m-1) / 2}$ if $m$ is odd. Then it is easy to show that $|f(x)|_{p} \leq \sqrt{|x|_{p}}$ for all $x$ implying that

$$
|f(x+y)-f(x)-f(y)|_{p} \leq \max \left\{\sqrt{|x|_{p}}, \sqrt{|y|_{p}}\right\}=: \varphi(x, y), \quad x, y \in \mathbb{Q} .
$$

Then (i) and (ii) are satisfied for $p$ (mainly since $\varphi\left(x / p^{n}, y / p^{n}\right) / p^{n}=p^{-n / 2}$ $\varphi(x, y))$, but also for $q$ instead of $p$ since $\varphi\left(x / q^{n}, y / q^{n}\right) / q^{n}=\varphi(x, y) / q^{n}$ and the estimate $\varphi_{q}\left(x / q^{n}\right) \leq(q-1) \sqrt{|x|_{p}}$ holds true.

Thus by Theorem 8 the sequence $\left(p^{n} f\left(x / p^{n}\right)\right)_{n \in \mathbb{N}_{0}}$ converges (resulting in an additive function). In this case the limit equals 0 for all $x$ since

$$
\left|p^{n} f\left(x / p^{n}\right)\right|_{p} \leq \frac{1}{p^{n}} \sqrt{\left|\frac{x}{p^{n}}\right|_{p}}=\frac{\sqrt{|x|_{p}}}{\sqrt{p}^{n}} \longrightarrow 0 \text { for } n \rightarrow \infty
$$

Finally let $x \neq 0$ and note that $\left|q^{n}\right|_{p}=1$ for all $n$. Then $|x|_{p}=\left|x / q^{n}\right|_{p}$ implying that $0 \neq f(x)=f\left(x / q^{n}\right)$. Thus the convergence of the sequence $\left(q^{n} f\left(x / q^{n}\right)\right)_{n \in \mathbb{N}_{0}}$ would imply the convergence of the sequence $\left(q^{n}\right)_{n \in \mathbb{N}_{0}}$. Then, however, the sequence $\left(q^{n+1}-q^{n}\right)_{n \in \mathbb{N}_{0}}$ would be a sequence converging to 0 . But $\left|q^{n+1}-q^{n}\right|_{p}=\left|q^{n}(q-1)\right|_{p}=|q-1|_{p} \neq 0, n \in \mathbb{N}_{0}$, a contradiction.

Funding Information Open access funding provided by University of Graz.

Open Access. This article is licensed under a Creative Commons Attribution 4.0 International License, which permits use, sharing, adaptation, distribution and reproduction in any medium or format, as long as you give appropriate credit to the original author(s) and the source, provide a link to the Creative Commons licence, and indicate if changes were made. The images or other third party material in this article are included in the article's Creative Commons licence, unless indicated otherwise in a credit line to the material. If material is not included in the article's Creative Commons licence and your intended use is not permitted by statutory regulation or exceeds the permitted use, you will need to obtain 
permission directly from the copyright holder. To view a copy of this licence, visit http:// creativecommons.org/licenses/by/4.0/.

Publisher's Note Springer Nature remains neutral with regard to jurisdictional claims in published maps and institutional affiliations.

\section{References}

[1] Forti, G.L.: An existence and stability theorem for a class of functional equations. Stochastica 4(1), 23-30 (1980)

[2] Forti, G.L.: Hyers-Ulam stability of functional equations in several variables. Aequ. Math. 50(1-2), 143-190 (1995)

[3] Forti, G.L., Schwaiger, J.: Stability of homomorphisms and completeness. C. R. Math. Acad. Sci. Soc. R. Can. 11(6), 215-220 (1989)

[4] Găvruţa, P.: A generalization of the Hyers-Ulam-Rassias stability of approximately additive mappings. J. Math. Anal. Appl. 184(3), 431-436 (1994)

[5] Hyers, D.H.: On the stability of the linear functional equation. Proc. Natl. Acad. Sci. USA 27, 222-224 (1941)

[6] Schwaiger, J.: 12. Remark, Report of Meeting, 25th ISFE. Aequ. Math. 35, 120-121 (1988)

[7] Schwaiger, J.: On the construction of the field of reals by means of functional equations and their stability and related topics. In: Developments in Functional Equations and Related Topics. Selected Papers Based on the Presentations at the 16th International Conference on Functional Equations and Inequalities, ICFEI, Będlewo, Poland, May 17-23, 2015. Springer, Cham, pp 275-295 (2017)

[8] Schwaiger, J.: Connections between the completion of normed spaces over nonArchimedean fields and the stability of the Cauchy equation. Ann. Math. Sil. 34, 151-163 (2020)

Harald Fripertinger and Jens Schwaiger Institute of Mathematics and Scientific Computing

University of Graz

Graz

Austria

e-mail: harald.fripertinger@uni-graz.at

Jens Schwaiger

e-mail: jens.schwaiger@uni-graz.at

Received: October 30, 2020

Revised: April 7, 2021

Accepted: April 9, 2021 